\title{
Ubiquitination in cancer stem cell: roles and targeted cancer therapy
}

\author{
Liu LIU, Shasha YIN, Charles BROBBEY, Wenjian GAN ${ }^{*}$ \\ Department of Biochemistry and Molecular Biology, Medical University of South Carolina, Charleston, SC 29425, USA. \\ *Correspondence: ganw@musc.edu \\ https://doi.org/10.37175/stemedicine.v1i3.37
}

\begin{abstract}
Cancer stem cells (CSCs) are a small subset of stem-like cells inside tumors, which possess abilities of unlimited self-renewal, differentiation and proliferation. Extensive studies have suggested that CSCs are one of the major drivers of tumor initiation, metastasis, relapse and therapeutic resistance. Several regulatory networks including transcriptional programs and various signaling pathways tightly control the stemness, proliferation and differentiation of CSCs. Emerging evidence has indicated that posttranslational modifications, especially ubiquitination, play a critical role in maintenance of CSC properties. In this review, we summarize current understandings on E3 ubiquitin ligase-mediated regulation of transcription factors and key signaling pathways involved in the regulation of CSCs, and discuss the strategy to target CSCs and E3 ubiquitin ligases for combating cancers.
\end{abstract}

Keywords: Cancer stem cell · Ubiquitination · E3 ligase · Cell signaling · Transcription factor

\section{Introduction}

Tumor heterogeneity is a well-known phenomenon that tumor cells derived from different tumors or the same tumor exhibit distinct genotypes and phenotypes, which increases the complexity of cancer diagnosis and treatment (1). Over the past several decades, a few models have been brought up to explain tumor heterogeneity including the predominant cancer stem cell (CSC) model (2), which states that among masses of cells inside a tumor, only a small portion of cells exhibit tumor initiation power (also termed tumor-initiating cells) (3).

In supporting of the CSC model, as early as 1800 s, Virchow and Cohnheim postulated that tumors would be rooted from the embryonic cells in the body of "embryonic rests" (4). In 1997, Bonnet and Dick provided the first evidence to demonstrate that CSCs exist in acute myeloid leukemia (AML). They found that a subset of patientderived AML cells were capable of initiating AML in immuno-suppressed mice (5). To date, CSCs have been isolated from breast, colon, ovary and many other solid tumors (6). Currently, it is broadly acknowledged that CSCs play critical roles in tumor initiation, metastasis, relapse and especially therapy resistance (7). CSCs could

Received: Feb 12, 2020; Accepted: Mar 3, 2020.

(c) The Author(s). 2020 This is an Open Access article distributed under the terms of the Creative Commons License (http://creativecommons.org/licenses/by/4.0/) which permits unrestricted use, distribution, and reproduction in any medium or format, provided the original work is properly cited. promote radioresistance and chemotherapy resistance via various mechanisms in different cancers, which provide CSCs with a survival advantage (8). Therefore, better understanding in CSC biology will facilitate targeting CSCs as a novel approach to combat cancers.

\section{Cancer Stem Cells}

CSCs are defined as a minority subset of cells within tumors, which have similar features as normal stem cells including self-renewal and differentiation, plus ability to form tumors (Figure 1) (9). CSCs may be originated from normal stem cells through accumulations of genetic alterations, which results in aberrant signaling and enables normal stem cells to obtain constitutively proliferative ability, leading to tumorigenesis (10). For example, introduction of the mutant p53 in breast cancer mouse model enhances breast cancer progression largely in part because of the expansion of mammary stem cells (11). CSCs may also arise from transformation of somatic cells through reprogramming network controlled by transcription factors. One of milestone findings in the stem cell research field is the generation of induced pluripotent stem cells (iPSCs) by Takahashi and Yamanaka in 2006. They found that over-expression of transcription factors Oct3/4, Sox2, c-Myc and Klf4 is sufficient to convert the mouse adult somatic cells into pluripotent embryoniclike cells under embryonic stem cell (ESC) culture conditions (12). Later studies have identified more critical pluripotency factors that can generate human iPSCs 
including Nanog (13, 14), LIN28 (15) and Glis1 (16) (Figure 1). These proteins are aberrantly activated in many cancers and CSCs. Notably, a recent study showed that overexpression of SOX2, POU3F2, OLIG2 and SALL2 transcription factors could convert the differentiated glioblastoma cells into fully tumorigenic CSCs (17).

Importantly, several core stemness signaling pathways including Notch, Wnt/ $\beta$-Catenin, Hedgehog, JAK/STAT and $\mathrm{NF}-\kappa \mathrm{B}$ pathways (Figure 1) are involved in the regulation of CSC properties (18). These pathways are aberrantly activated in CSCs and associated with CSCmediated tumorigenesis including leukemia, breast cancer, lung cancer and other solid tumors. For example, the activated form of STAT3 was significantly upregulated in breast CSC-like cells and inhibition of STAT3 resulted in decreased breast CSC proliferation and clonogenicity $(19,20)$. These stemness pathways cooperate with pluripotency factors to maintain CSC properties. Interestingly, the JAK/STAT3 signaling and OCT4 have a positive feedback loop: activation of STAT3 upregulates the mRNA levels of OCT4, while OCT4 could boost the activation of the JAK/STAT3 pathway $(21,22)$.

\section{Ubiquitination System}

Post-translational modifications (PTMs) are the key contributors to proteome diversity by conferring various functions on proteins. Ubiquitination is one of the most studied PTMs, which covalently conjugates the small protein ubiquitin (Ub) to the lysine residues (23). Ubiquitination process is a sequential enzymatic cascade consisting of three types of enzymes: ubiquitin-activating enzymes (termed E1s), ubiquitin-conjugating enzymes (termed E2s) and E3 ubiquitin ligases (termed E3s) (Figure 2) (24).
The E3s are the critical components responsible for the recognition of substrates and determination of substrate specificity. It is predicted that there are more than $600 \mathrm{E} 3 \mathrm{~s}$ in human, which can be classified into three major subfamilies: the RING (really interesting new gene) E3s, the HECT (homologous to the E6AP carboxyl terminus domain) E3s, and the RBR (RING-between-RING) E3s (25). These E3s are frequently deregulated in various human diseases and are emerging as attractive therapeutic targets (26).

Ubiquitination pathway regulates protein functions in many ways: marking them for proteasomal-mediated degradation, alteration of their cellular locations, and modulation of protein interactions (25). Ubiquitin can form seven types of poly-ubiquitin linkages on substrates through seven lysine residues (K6, K11, K27, K29, K33, K48 and K63), which serves as different signals to control protein functions. It is widely accepted that K11- and K48poly-ubiquitin linkages are the proteasome degradation markers, while K63-poly-ubiquitin linkage serves as a non-proteolytic modification in regulating protein activity, localization and signaling transduction (27). Therefore, ubiquitination pathway controls many fundamental biological processes such as replication, transcription and cell signaling transduction that regulate cell proliferation, apoptosis and tumorigenesis. In addition, ubiquitination pathway is a critical determinant of CSC cell fate, which regulates the activation of pluripotency factors and stemness signaling pathways (28).

\section{Regulation of CSC-Related Factors by Ubiquitination}

As the abundance of pluripotency factors is the key decider of cell fate, the expression of these factors may be regulated at DNA, RNA and protein levels. Notably, more than $80 \%$ of

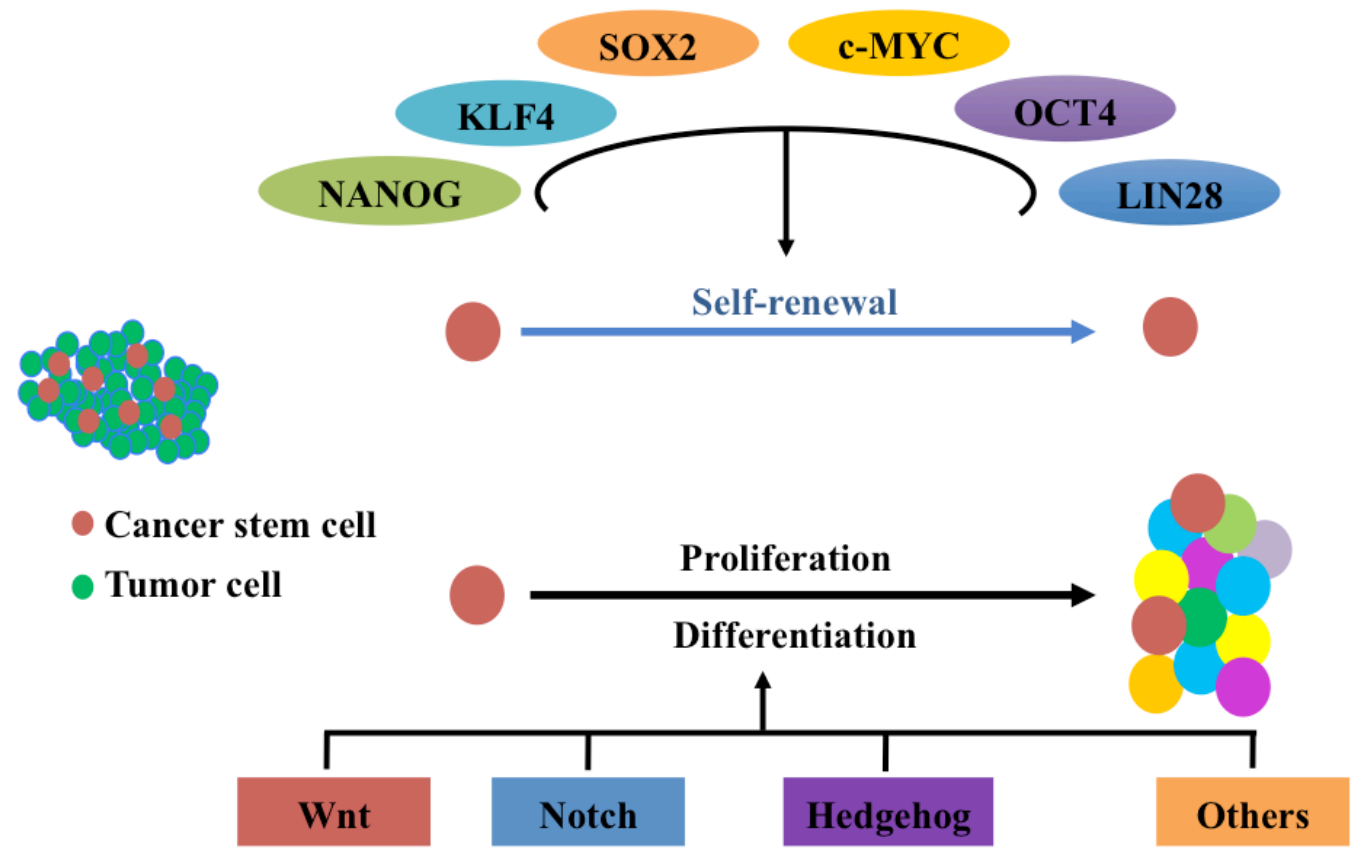

Figure 1. A schematic representation of key transcription factors and molecular signaling pathways involved in CSCs. 
proteins in cells are degraded by the ubiquitin-proteasome system (UPS) (29). A mass of evidences suggests that the UPS plays a critical role in controlling CSC properties via regulating the abundance of CSC-related proteins (Table 1).

\section{Oct3/4 (POU5F1)}

The proper protein levels of Oct3/4 determine distinct cell fate of ESCs. A study has found that down-regulation of Oct3/4 leads to loss of pluripotency, whereas less than two-folds up-regulation of Oct3/4 causes differentiation (30). Similarly, higher Oct4 expression promotes CSC expansion and tumorigenesis in breast cancer mouse model (31). In bladder cancer patient samples, higher expression levels of Oct4 are observed in more advanced cancers and contribute to poor survival (32). A few E3 ubiquitin ligases have been reported to control the Oct4 protein stability. WWP2, a HECT-type E3, interacts with and ubiquitinates Oct4 for $26 \mathrm{~S}$ proteasomal degradation upon the differentiation of ESCs (33). Itch, another HECT-type E3, catalyzes K63-linked poly-ubiquitination of Oct4, which enhances Oct4 protein stability. Depletion of Itch decreases Oct4 expression and significantly suppresses ESCs and iPSCs (34). A recent study has also found that the E3 ligase CHIP (carboxy terminus of HSP70interacting protein) is a novel partner of Oct4, which promotes Oct4 ubiquitination and degradation via the $26 \mathrm{~S}$ proteasome. Depletion of CHIP promotes breast CSCs, tumorigenesis and tumor metastasis in breast cancer mouse model. Importantly, a reverse correlation between the expression of CHIP and Oct4 was observed in breast cancer patients (35).

\section{Sox 2}

The stem cell-related transcription factor Sox 2 has participated in the maintenance of CSCs in a variety of cancers, including skin and breast cancers. Overexpression of Sox 2 enhances tumor initiation and metastasis (36). The E3 ligase WWP2 could target methylated Sox2 for ubiquitination and degradation, leading to cell differentiation (37). Interestingly, the Ube2s, an E2 ubiquitin-conjugating enzyme, mediates the K11-linked poly-ubiquitination of Sox2, resulting in its degradation by proteasome (38). More recently, CUL4A $\mathrm{A}^{\text {DET1-COP1, }}$ belonging to the Cullin-RING finger E3 family, was reported to catalyze Sox 2 poly-ubiquitination and degradation upon neural progenitor differentiation (39). These studies indicate that the E3 ligases of Sox 2 may govern cancer progression through regulating CSC functions.

\section{KLF4}

The role of the Krüppel-like factor (4KLF4) in cancers is context-dependent. It is a tumor suppressor and down-regulated in gastric cancer, liver cancer and lung cancer, whereas it is upregulated in breast cancer and osteosarcoma (40). CSC-enriched spheroid breast cancer cells display higher expression of KLF4. Consistently, overexpression of KLF4 increases CSC population and tumorigenesis in breast cancer (41). The abundance of KLF4 can be regulated by several E3 ubiquitin ligases. FBXO32, a member of SCF E3 ligase subfamily, suppresses breast tumorigenesis by promoting ubiquitination and degradation of KLF4 (42). Mule (Mcl-1 ubiquitin ligase E3), a HECT-type E3, could target KLF4 for degradation to promote entry into $\mathrm{S}$ phase and enhance proliferation of $\mathrm{T}$ cells (43). Interestingly, the protein levels of TRAF7 (tumor necrosis factor receptorassociated factor 7) are elevated in liver cancer, which is inversely correlated with the KLF4 expression. Further



Figure 2. A schematic representation of the ubiquitin-proteasome system. 
Table 1: The summary of E3 ligases in regulation of CSC-related proteins

\begin{tabular}{|c|c|c|c|}
\hline CSC-related protein & E3 ligase & Effect & Reference \\
\hline \multicolumn{4}{|l|}{ Transcription Factors } \\
\hline \multirow{3}{*}{ Oct3/4 (POU5F1) } & WWP2 & Degradation & 33 \\
\hline & ITCH & Degradation & 34 \\
\hline & CHIP & Degradation & 35 \\
\hline \multirow{2}{*}{ Sox 2} & WWP2 & Degradation & 37 \\
\hline & $\mathrm{COP} 1$ & Degradation & 39 \\
\hline \multirow{3}{*}{ KLF4 } & FBXO32 & Degradation & 42 \\
\hline & Mule & Degradation & 43 \\
\hline & TRAF7 & Degradation & 44 \\
\hline \multirow{4}{*}{$\mathrm{c}-\mathrm{Myc}$} & Fbw7 & Degradation & $48-51$ \\
\hline & $\beta$-TRCP & Stabilization & 52 \\
\hline & HectH9 & Enhanced activity & 53 \\
\hline & KCTD2 & Degradation & 54 \\
\hline \multirow{2}{*}{ Nanog } & SPOP & Degradation & 57 \\
\hline & FBXW8 & Degradation & 58 \\
\hline LIN28 & TRIM71 & Degradation & 61 \\
\hline \multicolumn{4}{|l|}{ Notch Signaling Pathway } \\
\hline \multirow{2}{*}{ Notch } & Fbw7 & Degradation & 64,66 \\
\hline & Itch & Degradation & 67 \\
\hline \multirow{2}{*}{$\begin{array}{l}\text { DLL1, DLL4, } \\
\text { JAG1, JAG2 }\end{array}$} & MIB1/MIB2 & Degradation & 68 \\
\hline & NEUR1/NEUR2 & Degradation & 68 \\
\hline \multicolumn{4}{|l|}{ Wnt Signaling Pathway } \\
\hline FZD, LRP6 & ZNRF3, RNF43 & Degradation & 71,72 \\
\hline \multirow{2}{*}{ DVL } & Itch & Degradation & 74 \\
\hline & NEDD4L & Degradation & 75 \\
\hline \multirow{2}{*}{$\beta$-catenin } & $\beta$-TRCP & Degradation & 76 \\
\hline & RNF146 & Degradation & 77 \\
\hline \multirow{2}{*}{ Axin } & Smurf1 & Inactivation & 78 \\
\hline & SIAH1 & Degradation & 79 \\
\hline APC & RNF61 & Degradation & 80 \\
\hline \multicolumn{4}{|c|}{ Hedgehog (Hh) Signaling Pathway } \\
\hline \multirow{2}{*}{ PTCH1 } & Smurf1, Smurf2 & & 84 \\
\hline & Itch & & 85 \\
\hline SMO & Unknown & Degradation & 86,87 \\
\hline \multirow{2}{*}{$\mathrm{SuFu}$} & Fbx117 & Degradation & 88 \\
\hline & Itch $/ \beta$-arrestin 2 & Inactivation & 89 \\
\hline GLI1 & $\beta$-TRCP & Degradation & 93 \\
\hline \multirow{2}{*}{ GLI2/3 } & $\beta$-TRCP & Partial degradation & 90,91 \\
\hline & SPOP & Degradation & 92 \\
\hline
\end{tabular}

study demonstrates that TRAF7 functions as an E3 ligase of KLF4 to promote KLF4 degradation and enhance cancer progression (44). Therefore, these E3 ligases may be responsible for the deregulation of KLF4 in various cancers.

\section{c-Myc}

The transcription factor c-Myc is a well-known oncogene that is overexpressed in more than $40 \%$ of human cancers. It controls all hallmarks of cancer including genome instability and sustaining proliferation (45). Extensive 
studies demonstrate that c-Myc serves as a key factor in the maintenance of CSCs. Inhibition of c-Myc leads to a decrease in CSC population by inducing senescence (46). UPS-mediated degradation of c-Myc represents a main mechanism for controlling its abundance. c-Myc has a short protein half-life, approximately 20-30 minutes (47). There are several E3 ubiquitin ligases responsible for the regulation of c-Myc expression. The E3 ligase Fbw7 (F-box and WD repeat domain-containing 7) could promote c-Myc degradation, which requires prior phosphorylation by glycogen synthase kinase 3 (GSK3) (48). In a chronic myeloid leukemia mouse model, knockout of Fbw 7 elevates c-Myc protein to re-initiate the cell cycle in leukemia-initiating cells (49-51). Interestingly, another F-box E3 ligase $\beta$-TRCP catalyzes K63-linked polyubiquitination on c-Myc, which stabilizes c-Myc protein by inhibiting Fbw7-mediated degradation (52). The HECT-domain E3 ligase HectH9 also catalyzes poly-ubiquitination of c-Myc with K63 linkage and consequently enhances c-Myc protein stability, promoting cell proliferation (53). The KCTD2 (potassium channel tetramerization domain-containing 2), a Cullin3-based E3, was also reported to promote degradation of c-Myc. Deletion of KCTD2 elevates c-Myc protein levels and confers CSC properties to glioma cells (54). Other E3 ligases including Skp2, TRIM32, Fbx29 and CHIP also control c-Myc stability.

\section{Nanog}

Nanog is upregulated in various cancers and CSCs and correlates with the stage and prognosis of cancers (55). Overexpression of Nanog enhances pluripotency and unlimited proliferation of CSCs (56). Recent studies have revealed that Nanog can be ubiquitinated and subsequently degraded by SPOP, a Cullin 3-based E3, leading to stemness loss of prostate cancer cells (57). The FBXW8 (F-box and WD40 domain-containing protein 8) induces stem cell differentiation by targeting Nanog for degradation (58).

\section{LIN28}

LIN28 is another reprogramming factor that can promote pluripotency by suppressing expression of microRNA let-7. LIN28 is an evolutionarily conserved RNA-binding protein that is highly expressed in ESCs and CSCs. It plays a critical role in the regulation of CSC pluripotency and is considered as a marker of CSCs. Depletion of LIN28 eradicates CSCs in ovarian cancer. Aberrant expression of LIN28A/LIN28B is observed in more aggressive cancers, and contributes to poor prognosis and drug resistance in certain cancer types $(59,60)$. TRIM71, a member of the tripartite-motif (TRIM) E3 family, negatively regulates LIN28B protein stability via ubiquitin-mediated proteasomal degradation, which leads to tumor suppression (61). However, it is largely unknown how LIN28 protein stability is regulated by other E3 ligases.

\section{Regulation of Stemness Signaling Pathways by}

\section{Ubiquitination}

PTMs are the heart of the signaling transduction, which can confer distinct functions to proteins in response to various environment changes (62). Ubiquitination, one of the most common PTMs, is a key player in controlling the activation of core stemness signaling pathways (Table 1).

\section{Notch Signaling Pathway}

The Notch signaling pathway is evolutionarily conserved from Drosophila to human. It has important roles in dictating development, tissue renewal, tumor initiation and metastasis. Canonical Notch signaling involves two adjacent cells expressing the Notch receptors and the ligands. Four Notch receptor paralogues (Notch1-4) and five Notch ligands (DLL1, DLL3, DLL4, JAG1 and JAG2) were identified in mammals (63). Both Notch receptors and ligands can be regulated by ubiquitination.

The Notch intracellular domain (NICD) contains a PEST domain (rich in proline, aspartic acid, serine and threonine residues) that can be recognized by E3 ligases. Upon activation, the NICD is promptly ubiquitinated and degraded by the E3 ubiquitin ligase Fbw7 in mammals and its ortholog SEL-10 in Caenorhabditis elegans (64). Constitutively active form of Notch with deletion of the PEST domain has been observed in some T-cell acute lymphoblastic leukemia (65). Moreover, loss of Fbw7 in neural stem cells (NSCs) elevates Notch protein levels, leading to imbalance between selfrenewal and differentiation, and finally aberrant brain development (66). Interestingly, the non-activated Notch is ubiquitinated with K29-linkage by Itch/AIP4 E3 ubiquitin ligase and subsequently subjected for lysosomal degradation (67). Studies have also showed that DLL1, DLL4, JAG1 and JAG2 undergo ubiquitination mediated by the RING family E3 ligases, MIB1/MIB2 and NEUR1/ NEUR2, which trigger ligand endocytosis (68). Despite advances in understanding the roles of ubiquitination in Notch signaling, it is unclear how these events contribute to $\mathrm{CSC}$ and cancer progression.

\section{Wnt Signaling Pathway}

Similar to the Notch pathway, the Wnt signaling pathway is another key cascade in controlling stemness and malignant growth. It is hyper-activated in different types of cancers particularly colorectal cancer. Notably, high Wnt activity is considered as a marker of colon cancer stem cells and promotes CSC expansion through upregulation of its downstream targets including CCND1, FOXM1, MYC and YAP/TAZ (69). The core components of canonical Wnt signaling pathway include receptor Frizzled (FZD), co-receptors LRP5/6, the scaffolding protein Dishevelled (DVL), the major effector $\beta$-catenin and destruction complex containing Axin, APC and GSK $3 \beta$ and casein kinase (CK1 $\alpha$ ) (70). These components can be regulated by the ubiquitination system, which contribute to the temporal and spatial regulation of Wnt signaling pathway activation.

Studies have showed that the zinc and ring finger 3 (ZNRF3) and ring finger 43 (RNF43) E3s target FZD 
and LRP6 for ubiquitination-dependent lysosomal degradation, leading to a decrease of FZD receptor at the cell surface $(71,72)$.

Multiple E3s are involved in regulation of the DVL protein stability. The Cullin-3 based E3 ligase, KLHL12, promotes DVL poly-ubiquitination and degradation in the absence of Wnt (73). Itch, a HECT-type E3, promotes ubiquitination and degradation of phosphorylated DVL depending on the PPXY motif and the DEP domain of DVL (74). The NEDD4L catalyzes the K6-, K27- and K29-linked atypical ubiquitin chains for targeting DVL degradation (75). Without the Wnt ligands, $\beta$-catenin is phosphorylated by the destruction complex and subsequently recognized and ubiquitinated by $\beta$-TRCP (76).

As a key determinant of the destruction complex, the expression of Axin is tightly controlled. Poly-ADPribosylated Axin can be recognized and ubiquitinated by the RING E3 ligase RNF146, leading to Axin degradation (77). Smurf1, a HECT-type E3, catalyzes non-proteolytic K29-linked ubiquitin chains on Axin and consequently impairs Axin interaction with LRP5/6, leading to shutdown of the Wnt signaling pathway. Interestingly, Itch-mediated ubiquitination of Axin is cell-cycle-dependent (78). More recently, a study has found that in the presence of Wnt stimulation, the seven in absentia homolog 1 (SIAH1) competes with GSK to bind and degrade Axin, providing a positive feedback activation of the Wnt signaling (79). Ubiquitination also governs the protein levels of APC to control the function of the destruction complex. Overexpression of MKRN1 E3 ligase induces ubiquitination and degradation of APC. In contrast, knockout of MKRN1 leads to accumulation of APC, which suppresses Wnt pathway activation and cell migration (80).

In addition to the ubiquitination-mediated protein turnover, APC and DVL also undergo K63-linked nonproteolytic poly-ubiquitination, while Axin can form K29linked poly-ubiquitination, all of which are important for the activation of Wnt signaling (81). As most of these E3 ubiquitin ligases are deregulated in cancers, these studies offer a possible explanation for the aberrant activation of Wnt signaling in CSCs and various cancers.

\section{Hedgehog (Hh) Signaling Pathway}

The controlled Hh signaling pathway is crucial for embryogenesis and proper organ growth. Its aberrant activation may promote tumorigenesis, tumor metastasis and drug resistance, which has been documented in leukemia, pancreatic cancer and many other solid tumors (82). Accumulating evidence demonstrates that the Hh pathway is critical for the maintenance and expansion of CSCs. The expression of the core Hh pathway components, including SMO, PTCH1, GLI2/3 and SuFu, is significantly upregulated in CSCs (83). Growing evidence suggests that deregulation of ubiquitination on these components is a predominate cause for the aberrancy of the Hh signaling pathway.

PTCH1 contains two PPXY motifs in the cytoplasmic C-tail, which mediates its interaction with Smurf1/2,
Nedd4, WWP2 and Itch that are HECT-type E3s. Upon Shh stimulation, the expression of Smurf1/2 is up-regulated and targets $\mathrm{PTCH} 1$ for degradation by catalyzing poly-ubiquitin chains with K48 and K63 linkages. Knockout of Smurf1/Smurf2 in mice impairs Shh-induced cerebellar organogenesis (84). In the absence of Hh signaling, Itch targets PTCH1 for ubiquitination and degradation (85). Although Nedd4 and WWP2 interact with PTCH1, they do not regulate PTCH1 stability.

SMO can be poly/mono-ubiquitinated, resulting in its degradation by lysosome or $26 \mathrm{~S}$ proteasome, which is inhibited by $\mathrm{Hh}$ stimulation $(86,87)$. However, the E3 ligases of SMO have not been identified yet.

Sufu is a tumor suppressor and a negative regulator of the Hh signaling pathway by sequestering GLI transcription factors in the cytoplasm. In response to Shh ligand, Sufu is ubiquitinated and degraded by E3 ligase Fbxl17 (F-box and leucine-rich repeat protein 17), leading to the activation of Hh signaling. Knockdown of Fbx117 leads to the accumulation of Sufu protein and reduction of tumor growth (88). SuFu also undergoes nonproteolytic K63-linked poly-ubiquitination catalyzing by the Itch/ $\beta$-arrestin 2 complex, which is inhibited by the $\mathrm{Hh}$ signaling. This event enhances $\mathrm{SuFu}$ interaction with GLI3 and keeps Hh signaling off, contributing to tumor suppression (89).

The ubiquitination modification of GLI transcription factors negatively regulates $\mathrm{Hh}$ pathway activation. In the absence of Hh, E3 ligase $\beta$-TRCP binds and ubiquitinates phosphorylated GLI2/3 that is mediated by kinases PKA, GSK $3 \beta$ and CK 1 . As a result, GLI2/3 are partially degraded to generate the repressor form $(90,91)$. In the presence of Hh, Cul3-based E3 ligase SPOP could target the activated full-length form of GLI2/3 for ubiquitination-mediated proteasomal degradation, which serves as a negative feedback regulation of $\mathrm{Hh}$ pathway activation (92). Interestingly, $\beta$-TRCP also targets GLI1 for complete proteolysis, without generation of the repressor form (93).

\section{Other Stemness Signaling Pathways}

The ubiquitination modification also has important functions in governing the activation of other stemness pathways including the NF- $\kappa \mathrm{B}, \mathrm{JAK} / \mathrm{STAT}$ and PI3K/ AKT pathways, which has been well discussed in other reviews (94-96).

\section{CSC-Targeting Therapies}

As CSCs are a key factor conferring drug-resistance, tumor recurrence and metastasis, targeting CSCs is becoming a potential and promising therapeutic approach. Growing evidence indicates that inactivation of CSCrelated transcription factors or signaling pathways can significantly suppress cancer progression and increase the cellular sensitivity to chemotherapy and radiotherapy in preclinical studies. To this end, many CSC-targeted agents have been developed and entered clinical trials (Table 2).

\section{Targeting Stemness Pathways}

Aberrant activation of stemness controlling pathways 
Table 2: The summary of drugs targeting the CSC-related proteins

\begin{tabular}{|c|c|c|c|}
\hline CSC-related proteins & Compound & Development phase & Reference \\
\hline \multirow{2}{*}{ Oct3/4 (POU5F1) } & KRIBB53 & Preclinical & 110 \\
\hline \multirow{3}{*}{ c-Myc } & MYCi361 & Preclinical & 111 \\
\cline { 2 - 4 } & $10058-F 4$ & Preclinical & 112 \\
\cline { 2 - 4 } & GSK525762 & Phase I, II & 113 \\
\hline \multirow{2}{*}{ LIN28 } & 1632 & Preclinical & 114 \\
\hline \multirow{3}{*}{ Notch } & LY3039478 & Phase I & 115 \\
\cline { 2 - 4 } & MK0752 & Phase I & 116 \\
\cline { 2 - 4 } & AL101 & Phase I, II & 117,118 \\
\hline FZD, & OMP-18R5 (Vantictumab) & Phase I & 119 \\
\hline DVL & NSC668036 & Preclinical & 120 \\
\hline \multirow{3}{*}{$\beta$-catenin } & PRI-724 & Phase I, II & 121 \\
\cline { 2 - 4 } & E7368 & Phase I & 122 \\
\cline { 2 - 4 } & BC-2059 & Phase I & 123 \\
\hline Axin & IWR-1-endo & Preclinical & 124 \\
\hline \multirow{2}{*}{ SMO } & Vismodegib & FDA approved & 125 \\
\cline { 2 - 4 } & Patidegib & Phase III & 126 \\
\cline { 2 - 4 } & Taladegib & Phase I, II & 127 \\
\hline
\end{tabular}

leads to the unlimited self-renewal and proliferation of CSCs, eventually tumorigenesis and drug resistance. Therefore, targeting these pathways might be a promising strategy to abrogate CSCs and cancers. One of the major Notch pathway inhibitors is the $\gamma$-secretase inhibitor (GSI), which comprises the formation of matured NICD by blocking proteolytic cleavages of Notch receptors. GSI has demonstrated strong anti-tumor activity in part by inducing apoptosis of CSCs (97). Combination of GSI with 5-fluorouracil enhances the inhibition on clonogenicity and tumorigenicity of CSCs (98). The Hh inhibitor vismodegib that targets SMO was used to clinically treat basal cell carcinoma and approved by the US Food and Drug Administration in 2012 (99). Many Wnt pathway inhibitors targeting FZD receptors, DVL and $\beta$-catenin are in early clinical trials (100).

\section{Targeting CSC-related Transcription Factors}

It is a historical challenge to directly target transcription factors for cancer therapy because the inhibitors targeting protein-DNA interaction are difficult to develop as druglike properties (101). However, emerging research evidence demonstrates that targeting the epigenetic signaling has the potential to be an effective approach for diminishing CSCs (102). Overexpression of JMJD3, a histone H3K27me3 demethylase, decreases OCT4 expression, which results in diminished CSCs and restarted tumor growth in breast cancer (103). BET inhibitor, JQ1, which competes BRD4 binding with acetylated histones at the enhancer of $c-M y c$, markedly decreases its expression, resulting in suppression of tumor growth in multiple cancer models $(104,105)$. Thus, inhibitors of epigenetic programming that suppress the expression of CSC-related transcription factors, might overcome drug resistance by abrogating CSCs.

\section{Targeting CSC-related E3 Ubiquitin Ligases}

As most of CSC-related E3 ubiquitin ligases are frequently defective in cancers, small molecules are needed to restore their expression and function. The milestone for targeting E3 ligases is the development of proteolysis-targeting chimeras (PROTACs) technology. Mechanically, PROTAC is a bifunctional molecule that bridges an E3 ubiquitin ligase and a target protein, promoting ubiquitination and degradation of the target protein by the hijacked E3 ligase (106). Notably, PROTACs including dBET1 and ARV-825 could hijack the E3 ubiquitin ligase cereblon to bind BRD4, resulting in more robust degradation of c-Myc, apoptosis induction and tumor growth compared to BET inhibitors $(107,108)$. It was also reported that oridonin, a natural diterpenoid compound, could induce Fbw7 expression and GSK3 activation, resulting in degradation of c-Myc (109). Therefore, reactivation of defective E3 ubiquitin ligases by either PROTAC possesses great potential for pursuing effective therapeutics.

\section{Conclusions and Perspectives}

All of the above illustrative examples highlight the role of E3s in control of CSC features and functions on cancer progression. However, knowledge on the ubiquitination and CSCs is far away to be completed. For example, E3 ubiquitin ligases controlling the stability of many CSCrelated proteins have not been identified or limited, including Nanog, LIN28 and FZD. Moreover, whether and how the non-proteolytic ubiquitination regulates 
CSC biology remains largely unknown. Protein-protein interaction analyses and genome-wide CRISPR screen will contribute to address these questions.

A growing amount of evidence suggests that E3s are potential targets for cancer therapy. Although it is at the early stage for the exploration of inhibitors or activators targeting E3 ubiquitin ligases, the results from preclinical studies and clinical trials thus far are highly promising and encouraging. Particularly, PROTACs offer an excellent opportunity to restore the function of E3 ligases for degrading many undruggable oncoprotein targets including transcription factors. For example, the cancerderived SPOP mutants fail to bind its substrates, such as Nanog and GLI2/3. Developing PROTACs for SPOP might restore its ability to target these oncoproteins. Therefore, better understanding the E3 ubiquitin ligases and CSCs will facilitate identification of novel therapeutic targets and approaches to combat cancers.

\section{Acknowledgements}

This work was supported in part by pilot research funding from Hollings Cancer Center's Cancer Center Support Grant P30 CA138313 at the Medical University of South Carolina, and by the grant R00CA207867 (W.G.) from National Cancer Institute.

\section{Conflict of interest}

The authors declare that they have no conflict of interest.

\section{References}

1. Fisher R, Pusztai L, Swanton C. Cancer heterogeneity: implications for targeted therapeutics. Br J Cancer. 2013;108(3):479-85.

2. Marusyk A, Polyak K. Tumor heterogeneity: causes and consequences. Biochim Biophys Acta. 2010;1805(1):105-17.

3. Kreso A, Dick JE. Evolution of the cancer stem cell model. Cell Stem Cell. 2014;14(3):275-91.

4. Capp JP. Cancer stem cells: from historical roots to a new perspective. J Oncol. 2019;2019:5189232.

5. Bonnet D, Dick JE. Human acute myeloid leukemia is organized as a hierarchy that originates from a primitive hematopoietic cell. Nat Med. 1997;3(7):730-7.

6. Bighetti-Trevisan RL, Sousa LO, Castilho RM, Almeida LO. Cancer stem cells: powerful targets to improve current anticancer therapeutics. Stem Cells Int. 2019;2019:9618065.

7. Ayob AZ, Ramasamy TS. Cancer stem cells as key drivers of tumour progression. J Biomed Sci. 2018;25(1):20.

8. Phi LTH, Sari IN, Yang YG, Lee SH, Jun N, Kim KS, et al. Cancer stem cells (CSCs) in drug eesistance and their therapeutic implications in cancer treatment. Stem Cells Int 2018;2018:5416923.

9. Batlle E, Clevers H. Cancer stem cells revisited. Nat Med 2017;23(10):1124-34

10. Plaks V, Kong N, Werb Z. The cancer stem cell niche: how essential is the niche in regulating stemness of tumor cells? Cell Stem Cell. 2015;16(3):225-38.

11. Shetzer Y, Molchadsky A, Rotter V. Oncogenic mutant p53 gain of function nourishes the vicious cycle of tumor development and cancer stem-cell formation. Cold Spring Harb Perspect Med. 2016;6(10).

12. Takahashi K, Yamanaka S. Induction of pluripotent stem cells from mouse embryonic and adult fibroblast cultures by defined factors. Cell. 2006;126(4):663-76.

13. Chambers I, Colby D, Robertson M, Nichols J, Lee S,
Tweedie S, et al. Functional expression cloning of Nanog, a pluripotency sustaining factor in embryonic stem cells. Cell. 2003;113(5):643-55.

14. Mitsui K, Tokuzawa $\mathrm{Y}$, Itoh H, Segawa K, Murakami M, Takahashi K, et al. The homeoprotein Nanog is required for maintenance of pluripotency in mouse epiblast and ES cells. Cell. 2003;113(5):631-42.

15. Yu J, Vodyanik MA, Smuga-Otto K, Antosiewicz-Bourget $\mathrm{J}$, Frane JL, Tian S, et al. Induced pluripotent stem cell lines derived from human somatic cells. Science. 2007;318(5858):1917-20.

16. Maekawa M, Yamaguchi K, Nakamura T, Shibukawa R, Kodanaka I, Ichisaka T, et al. Direct reprogramming of somatic cells is promoted by maternal transcription factor Glis1. Nature. 2011;474(7350):225-9.

17. Suva ML, Rheinbay E, Gillespie SM, Patel AP, Wakimoto H, Rabkin SD, et al. Reconstructing and reprogramming the tumor-propagating potential of glioblastoma stem-like cells. Cell. 2014;157(3):580-94.

18. Matsui WH. Cancer stem cell signaling pathways. Medicine (Baltimore). 2016;95(1 Suppl 1):S8-S19.

19. Zhou J, Wulfkuhle J, Zhang H, Gu P, Yang Y, Deng J, et al. Activation of the PTEN/mTOR/STAT3 pathway in breast cancer stem-like cells is required for viability and maintenance. Proc Natl Acad Sci U S A. 2007;104(41):16158-63.

20. Sherry MM, Reeves A, Wu JK, Cochran BH. STAT3 is required for proliferation and maintenance of multipotency in glioblastoma stem cells. Stem Cells. 2009;27(10):2383-92.

21. Yin X, Zhang BH, Zheng SS, Gao DM, Qiu SJ, Wu WZ, et al. Coexpression of gene Oct4 and Nanog initiates stem cell characteristics in hepatocellular carcinoma and promotes epithelial-mesenchymal transition through activation of Stat3/Snail signaling. J Hematol Oncol. 2015;8:23.

22. Do DV, Ueda J, Messerschmidt DM, Lorthongpanich C, Zhou $Y$, Feng B, et al. A genetic and developmental pathway from STAT3 to the OCT4-NANOG circuit is essential for maintenance of ICM lineages in vivo. Genes Dev. 2013;27(12):1378-90.

23. Hershko A, Ciechanover A. The ubiquitin system. Annu Rev Biochem. 1998;67:425-79.

24. Skaar JR, Pagan JK, Pagano M. SCF ubiquitin ligase-targeted therapies. Nat Rev Drug Discov. 2014;13(12):889-903.

25. Zheng N, Shabek N. Ubiquitin Ligases: Structure, Function, and Regulation. Annu Rev Biochem. 2017;86:129-57.

26. Wang D, Ma L, Wang B, Liu J, Wei W. E3 ubiquitin ligases in cancer and implications for therapies. Cancer Metastasis Rev. 2017;36(4):683-702.

27. Akutsu M, Dikic I, Bremm A. Ubiquitin chain diversity at a glance. J Cell Sci. 2016;129(5):875-80

28. Kang B, Sun XH. Regulation of cancer stem cells by RING finger ubiquitin ligases. Stem Cell Investig. 2014;1:5.

29. Amm I, Sommer T, Wolf DH. Protein quality control and elimination of protein waste: the role of the ubiquitin-proteasome system. Biochim Biophys Acta. 2014;1843(1):182-96.

30. Niwa H, Miyazaki J, Smith AG. Quantitative expression of Oct-3/4 defines differentiation, dedifferentiation or selfrenewal of ES cells. Nat Genet. 2000;24(4):372-6.

31. Kim RJ, Nam JS. OCT4 expression enhances features of cancer stem cells in a mouse model of breast cancer. Lab Anim Res. 2011;27(2):147-52.

32. Chang CC, Shieh GS, Wu P, Lin CC, Shiau AL, Wu CL. Oct-3/4 expression reflects tumor progression and regulates motility of bladder cancer cells. Cancer Res. 2008;68(15):6281-91.

33. Xu H, Wang W, Li C, Yu H, Yang A, Wang B, et al. WWP2 promotes degradation of transcription factor OCT4 in human embryonic stem cells. Cell Res. 2009;19(5):561-73.

34. Liao B, Zhong $\mathrm{X}, \mathrm{Xu} \mathrm{H}, \mathrm{Xiao} \mathrm{F}$, Fang Z, Gu J, et al. Itch, an E3 ligase of Oct4, is required for embryonic stem cell self-renewal and pluripotency induction. J Cell Physiol. 2013;228(7):1443-51.

35. Cho Y, Kang HG, Kim SJ, Lee S, Jee S, Ahn SG, et al. Post-translational modification of OCT4 in breast cancer 
tumorigenesis. Cell Death Differ. 2018;25(10):1781-95.

36. Mamun MA, Mannoor K, Cao J, Qadri F, Song X. SOX2 in cancer stemness: tumor malignancy and therapeutic potentials. J Mol Cell Biol. 2020;12(2):85-98.

37. Fang L, Zhang L, Wei W, Jin X, Wang P, Tong $Y$, et al. A methylation-phosphorylation switch determines Sox2 stability and function in ESC maintenance or differentiation. Mol Cell. 2014;55(4):537-51.

38. Wang J, Zhang Y, Hou J, Qian X, Zhang H, Zhang Z, et al. Ube2s regulates Sox2 stability and mouse ES cell maintenance. Cell Death Differ. 2016;23(3):393-404.

39. Cui CP, Zhang Y, Wang C, Yuan F, Li H, Yao Y, et al. Dynamic ubiquitylation of Sox2 regulates proteostasis and governs neural progenitor cell differentiation. Nat Commun. 2018;9(1):4648.

40. Wang L, Shen F, Stroehlein JR, Wei D. Context-dependent functions of KLF4 in cancers: could alternative splicing isoforms be the key? Cancer Lett. 2018;438:10-6.

41. Yu F, Li J, Chen H, Fu J, Ray S, Huang S, et al. Kruppel-like factor 4 (KLF4) is required for maintenance of breast cancer stem cells and for cell migration and invasion. Oncogene. 2011;30(18):2161-72.

42. Zhou H, Liu Y, Zhu R, Ding F, Wan Y, Li Y, et al. FBXO32 suppresses breast cancer tumorigenesis through targeting KLF4 to proteasomal degradation. Oncogene. 2017;36(23):3312-21.

43. Hao Z, Sheng Y, Duncan GS, Li WY, Dominguez C, Sylvester $\mathrm{J}$, et al. K48-linked KLF4 ubiquitination by E3 ligase Mule controls T-cell proliferation and cell cycle progression. Nat Commun. 2017;8:14003.

44. He H, Wu Z, Li S, Chen K, Wang D, Zou H, et al. TRAF7 enhances ubiquitin-degradation of KLF4 to promote hepatocellular carcinoma progression. Cancer Lett. 2020;469:380-9.

45. Chen $H$, Liu H, Qing G. Targeting oncogenic Myc as a strategy for cancer treatment. Signal Transduct Target Ther. 2018;3:5.

46. Yang A, Qin S, Schulte BA, Ethier SP, Tew KD, Wang GY. MYC Inhibition Depletes Cancer Stem-like Cells in TripleNegative Breast Cancer. Cancer Res. 2017;77(23):6641-50.

47. Gregory MA, Hann SR. c-Myc proteolysis by the ubiquitinproteasome pathway: stabilization of c-Myc in Burkitt's lymphoma cells. Mol Cell Biol. 2000;20(7):2423-35.

48. King B, Trimarchi T, Reavie L, Xu L, Mullenders J, Ntziachristos $P$, et al. The ubiquitin ligase FBXW7 modulates leukemia-initiating cell activity by regulating MYC stability. Cell. 2013;153(7):1552-66.

49. Thompson BJ, Jankovic V, Gao J, Buonamici S, Vest $A$, Lee JM, et al. Control of hematopoietic stem cell quiescence by the E3 ubiquitin ligase Fbw7. J Exp Med. 2008;205(6):1395-408.

50. Reavie L, Della Gatta G, Crusio K, Aranda-Orgilles B, Buckley SM, Thompson B, et al. Regulation of hematopoietic stem cell differentiation by a single ubiquitin ligase-substrate complex. Nat Immunol. 2010;11(3):207-15.

51. Takeishi S, Matsumoto A, Onoyama I, Naka K, Hirao A Nakayama KI. Ablation of Fbxw7 eliminates leukemiainitiating cells by preventing quiescence. Cancer Cell. 2013;23(3):347-61.

52. Popov N, Schulein C, Jaenicke LA, Eilers M. Ubiquitylation of the amino terminus of Myc by SCF(beta-TrCP) antagonizes SCF(Fbw7)-mediated turnover. Nat Cell Biol. 2010;12(10):973-81.

53. Adhikary S, Marinoni F, Hock A, Hulleman E, Popov N, Beier $\mathrm{R}$, et al. The ubiquitin ligase $\mathrm{HectH} 9$ regulates transcriptional activation by Myc and is essential for tumor cell proliferation. Cell. 2005;123(3):409-21.

54. Kim EJ, Kim SH, Jin X, Jin X, Kim H. KCTD2, an adaptor of Cullin3 E3 ubiquitin ligase, suppresses gliomagenesis by destabilizing c-Myc. Cell Death Differ. 2017;24(4):649-59.

55. Meng HM, Zheng $P$, Wang XY, Liu C, Sui HM, Wu SJ, et al. Over-expression of Nanog predicts tumor progression and poor prognosis in colorectal cancer. Cancer Biol Ther. 2010;9(4):295-302.

56. Wang ML, Chiou SH, Wu CW. Targeting cancer stem cells: emerging role of Nanog transcription factor. Onco Targets Ther. 2013:6:1207-20.

57. Zhang J, Chen M, Zhu Y, Dai X, Dang F, Ren J, et al. SPOP Promotes Nanog Destruction to Suppress Stem Cell Traits and Prostate Cancer Progression. Dev Cell. 2019;48(3):329-44 e5.

58. Kim SH, Kim MO, Cho YY, Yao K, Kim DJ, Jeong $\mathrm{CH}$, et al. ERK1 phosphorylates Nanog to regulate protein stability and stem cell self-renewal. Stem Cell Res. 2014;13(1):1-11.

59. Balzeau J, Menezes MR, Cao S, Hagan JP. The LIN28/let-7 Pathway in Cancer. Front Genet. 2017;8:31.

60. Zhou J, Ng SB, Chng WJ. LIN28/LIN28B: an emerging oncogenic driver in cancer stem cells. Int J Biochem Cell Biol. 2013;45(5):973-8.

61. Lee SH, Cho S, Kim MS, Choi K, Cho JY, Gwak HS, et al. The ubiquitin ligase human TRIM71 regulates let-7 microRNA biogenesis via modulation of Lin28B protein. Biochim Biophys Acta. 2014;1839(5):374-86.

62. Karve TM, Cheema AK. Small changes huge impact: the role of protein posttranslational modifications in cellular homeostasis and disease. J Amino Acids. 2011;2011:207691.

63. Guruharsha KG, Kankel MW, Artavanis-Tsakonas S. The Notch signalling system: recent insights into the complexity of a conserved pathway. Nat Rev Genet. 2012;13(9):654-66.

64. Fryer CJ, White JB, Jones KA. Mastermind recruits CycC:CDK8 to phosphorylate the Notch ICD and coordinate activation with turnover. Mol Cell. 2004;16(4):509-20.

65. Haydu JE, De Keersmaecker K, Duff MK, Paietta E, Racevskis $\mathrm{J}$, Wiernik $\mathrm{PH}$, et al. An activating intragenic deletion in NOTCH1 in human T-ALL. Blood. 2012;119(22):5211-4.

66. Matsumoto A, Onoyama I, Sunabori T, Kageyama R, Okano $\mathrm{H}$, Nakayama KI. Fbxw7-dependent degradation of Notch is required for control of "stemness" and neuronalglial differentiation in neural stem cells. J Biol Chem. 2011;286(15):13754-64

67. Qiu L, Joazeiro C, Fang N, Wang HY, Elly C, Altman Y, et al. Recognition and ubiquitination of Notch by Itch, a hect-type E3 ubiquitin ligase. J Biol Chem. 2000;275(46):35734-7.

68. Weinmaster G, Fischer JA. Notch ligand ubiquitylation: what is it good for? Dev Cell. 2011;21(1):134-44

69. de Sousa EMF, Vermeulen L. Wnt signaling in cancer stem cell biology. Cancers (Basel). 2016;8(7).

70. Komiya Y, Habas R. Wnt signal transduction pathways. Organogenesis. 2008;4(2):68-75.

71. Hao HX, Xie Y, Zhang Y, Charlat O, Oster E, Avello M, et al. ZNRF3 promotes Wnt receptor turnover in an R-spondinsensitive manner. Nature. 2012;485(7397):195-200.

72. Koo BK, Spit M, Jordens I, Low TY, Stange DE, van de Wetering M, et al. Tumour suppressor RNF43 is a stem-cell E3 ligase that induces endocytosis of Wnt receptors. Nature. 2012;488(7413):665-9.

73. Funato $Y$, Terabayashi T, Sakamoto R, Okuzaki D, Ichise $H$, Nojima $H$, et al. Nucleoredoxin sustains Wnt/beta-catenin signaling by retaining a pool of inactive dishevelled protein. Curr Biol. 2010;20(21):1945-52

74. Wei W, Li M, Wang J, Nie F, Li L. The E3 ubiquitin ligase ITCH negatively regulates canonical Wnt signaling by targeting dishevelled protein. Mol Cell Biol. 2012;32(19):3903-12.

75. Ding $Y$, Zhang $Y, X u C$, Tao $Q H$, Chen YG. HECT domaincontaining E3 ubiquitin ligase NEDD4L negatively regulates Wnt signaling by targeting dishevelled for proteasomal degradation. J Biol Chem. 2013;288(12):8289-98.

76. Liu C, Kato Y, Zhang Z, Do VM, Yankner BA, He X. betaTrcp couples beta-catenin phosphorylation-degradation and regulates Xenopus axis formation. Proc Natl Acad Sci U S A. 1999;96(11):6273-8. 
77. Zhang Y, Liu S, Mickanin C, Feng Y, Charlat O, Michaud GA, et al. RNF146 is a poly(ADP-ribose)-directed E3 ligase that regulates axin degradation and Wnt signalling. Nat Cell Biol. 2011;13(5):623-9.

78. Fei C, He X, Xie S, Miao H, Zhou Z, Li L. Smurf1-mediated axin ubiquitination requires Smurf1 $\mathrm{C} 2$ domain and is cel cycle-dependent. J Biol Chem. 2014;289(20):14170-7.

79. Ji L, Jiang B, Jiang X, Charlat O, Chen A, Mickanin C, et al The SIAH E3 ubiquitin ligases promote Wnt/beta-catenin signaling through mediating Wnt-induced Axin degradation. Genes Dev. 2017;31(9):904-15.

80. Lee HK, Lee EW, Seo J, Jeong M, Lee SH, Kim SY, et al Ubiquitylation and degradation of adenomatous polyposis coli by MKRN1 enhances Wnt/beta-catenin signaling. Oncogene. 2018;37(31):4273-86.

81. Tauriello DV, Maurice MM. The various roles of ubiquitin in Wnt pathway regulation. Cell Cycle. 2010;9(18):3700-9.

82. Wicking $\mathrm{C}$, McGlinn $\mathrm{E}$. The role of hedgehog signalling in tumorigenesis. Cancer Lett. 2001;173(1):1-7.

83. Cochrane CR, Szczepny A, Watkins DN, Cain JE. Hedgehog Signaling in the Maintenance of Cancer Stem Cells. Cancers (Basel). 2015;7(3):1554-85.

84. Yue S, Tang LY, Tang Y, Tang Y, Shen QH, Ding J, et al Requirement of Smurf-mediated endocytosis of Patched1 in sonic hedgehog signal reception. Elife. 2014;3.

85. Chen XL, Chinchilla P, Fombonne J, Ho L, Guix C, Keen JH, et al. Patched-1 proapoptotic activity is downregulated by modification of K1413 by the E3 ubiquitin-protein ligase Itchy homolog. Mol Cell Biol. 2014;34(20):3855-66.

86. Xia R, Jia H, Fan J, Liu Y, Jia J. USP8 promotes smoothened signaling by preventing its ubiquitination and changing its subcellular localization. PLoS Biol. 2012;10(1):e1001238.

87. Li S, Chen Y, Shi Q, Yue T, Wang B, Jiang J. Hedgehogregulated ubiquitination controls smoothened trafficking and cell surface expression in Drosophila. PLoS Biol. 2012;10(1):e1001239.

88. Raducu M, Fung E, Serres S, Infante $P$, Barberis A, Fischer $\mathrm{R}$, et al. SCF (Fbxl17) ubiquitylation of Sufu regulates Hedgehog signaling and medulloblastoma development. EMBO J. 2016;35(13):1400-16.

89. Infante $\mathrm{P}$, Faedda $\mathrm{R}$, Bernardi F, Bufalieri F, Lospinoso Severini L, Alfonsi R, et al. Itch/beta-arrestin2-dependent non-proteolytic ubiquitylation of SuFu controls Hedgehog signalling and medulloblastoma tumorigenesis. Nat Commun. 2018;9(1):976.

90. Bhatia N, Thiyagarajan S, Elcheva I, Saleem M, Dlugosz A, Mukhtar $\mathrm{H}$, et al. Gli2 is targeted for ubiquitination and degradation by beta-TrCP ubiquitin ligase. J Biol Chem. 2006;281(28):19320-6.

91. Wang B, Li Y. Evidence for the direct involvement of \{beta\} TrCP in Gli3 protein processing. Proc Natl Acad Sci U S A. 2006;103(1):33-8.

92. Zhang Q, Shi Q, Chen Y, Yue T, Li S, Wang B, et al. Multiple Ser/Thr-rich degrons mediate the degradation of $\mathrm{Ci} / \mathrm{Gli}$ by the Cul3-HIB/SPOP E3 ubiquitin ligase. Proc Natl Acad Sci U S A. 2009;106(50):21191-6.

93. Deng W, Vanderbilt DB, Lin CC, Martin KH, Brundage KM, Ruppert JM. SOX9 inhibits beta-TrCP-mediated protein degradation to promote nuclear GLI1 expression and cancer stem cell properties. J Cell Sci. 2015;128(6):1123-38.

94. Hatakeyama S. Ubiquitin-mediated regulation of JAKSTAT signaling in embryonic stem cells. JAKSTAT. 2012;1(3):168-75

95. Yang WL, Wu CY, Wu J, Lin HK. Regulation of Akt signaling activation by ubiquitination. Cell Cycle. 2010;9(3):487-97.

96. Chen ZJ. Ubiquitin signalling in the NF-kappaB pathway. Nat Cell Biol. 2005;7(8):758-65.

97. Shih le M, Wang TL. Notch signaling, gammasecretase inhibitors, and cancer therapy. Cancer Res. 2007;67(5):1879-82.

98. Capodanno Y, Buishand FO, Pang LY, Kirpensteijn J, Mol JA,
Argyle DJ. Notch pathway inhibition targets chemoresistant insulinoma cancer stem cells. Endocr Relat Cancer. 2018;25(2):131-44.

99. Proctor AE, Thompson LA, O'Bryant CL. Vismodegib: an inhibitor of the Hedgehog signaling pathway in the treatment of basal cell carcinoma. Ann Pharmacother. 2014;48(1):99-106.

100. Krishnamurthy N, Kurzrock R. Targeting the Wnt/beta-catenin pathway in cancer: Update on effectors and inhibitors. Cancer Treat Rev. 2018;62:50-60.

101. Darnell JE, Jr. Transcription factors as targets for cancer therapy. Nat Rev Cancer. 2002;2(10):740-9.

102. Toh TB, Lim JJ, Chow EK. Epigenetics in cancer stem cells. Mol Cancer. 2017;16(1):29.

103. Xun J, Wang D, Shen L, Gong J, Gao R, Du L, et al JMJD3 suppresses stem cell-like characteristics in breast cancer cells by downregulation of Oct4 independently of its demethylase activity. Oncotarget. 2017;8(13):21918-29.

104. Delmore JE, Issa GC, Lemieux ME, Rahl PB, Shi J, Jacobs $\mathrm{HM}$, et al. BET bromodomain inhibition as a therapeutic strategy to target c-Myc. Cell. 2011;146(6):904-17.

105. Mertz JA, Conery AR, Bryant BM, Sandy P, Balasubramanian $S$, Mele DA, et al. Targeting MYC dependence in cancer by inhibiting BET bromodomains. Proc Natl Acad Sci U S A. 2011;108(40):16669-74.

106. Paiva SL, Crews CM. Targeted protein degradation: elements of PROTAC design. Curr Opin Chem Biol. 2019;50:111-9.

107. Winter GE, Buckley DL, Paulk J, Roberts JM, Souza A, DhePaganon S, et al. DRUG DEVELOPMENT. Phthalimide conjugation as a strategy for in vivo target protein degradation. Science. 2015;348(6241):1376-81.

108. Lu J, Qian Y, Altieri M, Dong H, Wang J, Raina K, et al. Hijacking the E3 Ubiquitin Ligase Cereblon to Efficiently Target BRD4. Chem Biol. 2015;22(6):755-63.

109. Huang HL, Weng HY, Wang LQ, Yu CH, Huang QJ, Zhao PP, et al. Triggering Fbw7-mediated proteasomal degradation of c-Myc by oridonin induces cell growth inhibition and apoptosis. Mol Cancer Ther. 2012;11(5):1155-65.

110. Jung J, Kim Y, Song J, Yoon YJ, Kim DE, Kim JA, et al. KRIBB53 binds to OCT4 and enhances its degradation through the proteasome, causing apoptotic cell death of OCT4-positive testicular germ cell tumors. Carcinogenesis. 2018;39(6):838-49.

111. Han H, Jain AD, Truica MI, Izquierdo-Ferrer J, Anker JF, Lysy B, et al. Small-Molecule MYC Inhibitors Suppress Tumor Growth and Enhance Immunotherapy. Cancer Cell. 2019;36(5):483-97 e15.

112. Huang MJ, Cheng YC, Liu CR, Lin S, Liu HE. A smallmolecule c-Myc inhibitor, 10058-F4, induces cell-cycle arrest, apoptosis, and myeloid differentiation of human acute myeloid leukemia. Exp Hematol. 2006;34(11):1480-9.

113. Matkar S, Sharma P, Gao S, Gurung B, Katona BW, Liao J, et al. An epigenetic pathway regulates sensitivity of breast cancer cells to HER2 inhibition via FOXO/c-Myc axis. Cancer Cell. 2015;28(4):472-85.

114. Roos M, Pradere U, Ngondo RP, Behera A, Allegrini S, Civenni $G$, et al. A small-molecule inhibitor of Lin28. ACS Chem Biol. 2016;11(10):2773-81.

115. Massard C, Azaro A, Soria JC, Lassen U, Le Tourneau C, Sarker D, et al. First-in-human study of LY3039478, an oral Notch signaling inhibitor in advanced or metastatic cancer. Ann Oncol. 2018;29(9):1911-7.

116. Cook N, Basu B, Smith DM, Gopinathan A, Evans J, Steward WP, et al. A phase I trial of the gamma-secretase inhibitor MK-0752 in combination with gemcitabine in patients with pancreatic ductal adenocarcinoma. $\mathrm{Br} \mathrm{J}$ Cancer. 2018;118(6):793-801.

117. A phase I study of AL101, a pan-NOTCH inhibitor, in patients (pts) with locally advanced or metastatic solid tumors J Clin Oncol. 2018;36:15_suppl,2515.

118. ACCURACY: phase $(P) 2$ trial of AL101, a pan-Notch inhibitor, 
inhibitor, in patients (pts) with recurrent/metastatic (R/M) adenoid cystic carcinoma (ACC) with Notch activating mutations (Notchact mut). J Clin Oncol. 2019;37:15 suppl,TPS6098.

119. Smith DC, Rosen LS, Chugh R, et al. First-in-human evaluation of the human monoclonal antibody vantictumab (OMP-18r5; anti-Frizzled) targeting the WNT pathway in a phase I study for patients with advanced solid tumors. J Clin Oncol. 2013;31:15_suppl,2540.

120. Shan J, Shi DL, Wang J, Zheng J. Identification of a specific inhibitor of the dishevelled PDZ domain. Biochemistry. 2005;44(47):15495-503.

121. Kimura K, Ikoma A, Shibakawa M, Shimoda S, Harada K, Saio M, et al. Safety, tolerability, and preliminary efficacy of the anti-fibrotic small molecule PRI-724, a CBP/beta-catenin inhibitor, in patients with hepatitis $C$ virus-related virrhosis: a single-center, open-label, dose escalation phase 1 trial. EBioMedicine. 2017;23:79-87.

122. A study of E-7386 in participants with advanced solid tumor including colorectal cancer (CRC) (NCT03833700, 2019). Available online at: https://clinicaltrials.gov/ct2/show/ NCT03833700
123. Phase I, open-label, non-randomized study to evaluate safety of BC2059 (NCT03459469, 2018). Available online at: https://clinicaltrials.gov/ct2/show/NCT03459469

124. Lu J, Ma Z, Hsieh JC, Fan CW, Chen B, Longgood JC, et al. Structure-activity relationship studies of small-molecule inhibitors of Wnt response. Bioorg Med Chem Lett. 2009;19(14):3825-7.

125. Axelson M, Liu K, Jiang X, He K, Wang J, Zhao H, et al. U.S. Food and drug administration approval: vismodegib for recurrent, locally advanced, or metastatic basal cell carcinoma. Clin Cancer Res. 2013;19(9):2289-93.

126. Study of patidegib topical gel, $2 \%$, for the reduction of disease burden of persistently developing basal cell carcinomas (BCCs) in subjects with basal cell nevus syndrome (Gorlin Syndrome) (NCT03703310, 2018). Available online at: https://clinicaltrials.gov/ct2/show/ NCT03703310

127. Taladegib, paclitaxel, carboplatin, and radiation therapy in treating patients with localized esophageal or gastroesophageal junction cancer (NCT02530437, 2015). Available online at: https://clinicaltrials.gov/ct2/show/ NCT02530437. 